\title{
Gender markers in the space of a Russian university and their role in assessing the quality students' life (on the example of regional universities)
}

\author{
Anastasia Babaeva*, Artem Kluev, Julia Marinina, Anna Merzlyakova, and Julia Shlykova
}

Kozma Minin Nizhny Novgorod State Pedagogical University, Ulyanov str, 1, 603000 Nizhny

Novgorod, Russia

\begin{abstract}
The article is devoted to the analysis of subjective well-being which is concerned as a constituent element of the integral concept of the quality of life. Theoretical conclusions of the study are based on empirical data from sociological research. The study focuses on Russian students and their well-being in Russian educational environment. The authors examine the influence of gender aspects on the students' comfort level. Gender markers which function in business and personal communication at the university are investigated in vertical and horizontal cross-sections. Some peculiarities in the assessments given by Russian students on the subject of gender-sensitive social practices are noted. The main factors that determine the assessment of gender markers presence at the university are revealed. The lack of formed gender competence among Russian students is also revealed. The influence of gender markers on student satisfaction with studying process at the university is determined.
\end{abstract}

\section{Introduction}

The concept of the quality of life, which the UN has put as the basis for comparing and assessing the socio-economic development of countries, is integrative. It includes indicators that reflect physical, mental, emotional and social health of a person. The World Health Organization (WHO) proposes to interpret the term "quality of life" as an individuals" perception of their position in life in the context of the culture and value systems in which they live and in relation to their goals, expectations, standards and concerns". Thus, this concept includes the following indicators: living standards, level of governmental and social support of individual interests and goals, compliance with expectations. Some countries consider as part of the concept the opportunity to demonstrate various kinds of activity and enjoy them [1].

The complexity of using the concept of quality of life (and its derivatives) is determined by the fact that there is no single, generally accepted interpretation of it.

However, a set of fundamental indicators that appear in the UN reports allows us to make some conclusions: 1) consideration of the quality of life of a country is directly

${ }^{*}$ Corresponding author: dff1890@yandex.ru 
related to its prospects for socio-economic development; 2) the study of the quality of life raises the problem of national security. The phenomenon of national security is associated with the resistance of socio-economic, political and cultural systems to destabilizing external and internal impacts [2].

The structure of the concept in question includes, first of all, objective indicators: life expectancy, absence of threats to life and health, level of unemployment, volume of consumption of goods and services, access to cultural values and education, etc. These are the metrics that are quantified and described. The key indicators here are the volume and dynamics of gross domestic product and the human development index (HDI). The second block of indicators consists of subjective factors - the index of happiness, or satisfaction with one's life. These are qualitative indicators, they are more difficult to analyze, but in combination with them, the actual data reflect the real picture of the quality of life [3].

The purpose of this report is to analyze the subjective factor, more specifically satisfaction with the life of a particular social group - student youth. We focus on the degree of comfort of learning process for higher education students. The study also takes in consideration the functioning of gender markers in university environment.

In this regard, the study of gender markers in the environment of Russian university raisis the range of problems associated with a) the demographic situation (the issue of Russian youth reproductive health and their reproductive behavior); b) social relations and the level of conflict in society (lack of gender asymmetry and harmonious relations in the educational environment); c) cognition of the world and personal development, carried out through the institution of education (equal access to different degrees of education in various specialties, regardless of gender).

Such studies are becoming more and more important for several reasons. Firstly, the modern stage of social development is characterized by the transition from a production economy to a knowledge economy. This means that knowledge, values and experience are becoming the leading factor in socialization. So education can be interpreted as a key to the future, the way to produce a new human format. Everything is important here: the content of the courses (what is broadcast), the teaching method (how it is broadcast), the equipment of the classrooms and the organization of classes (where it is broadcast). Researchers shouldn't lose sight of extracurricular communication principles and general principals of learning process organization.

The role of educational institutions in modern Western society, which includes Russia, is also increasing because traditional institutions - the church and the family are loosing their importance. At the same time the concept of lifelong education is gaining popularity. As a result, we get a social institution that accompanies a person throughout his life. Individual's existence largely depends on what he or she receives at University: what knowledge and values. It also depends on how comfortable an individual feels at University as well.

Secondly, there is growing interest in the study of the gender aspects of educational practices, it is due to the increasing technocratization and informatization of social practices [4].

New spheres and forms of employment are emerging. For example, to apply for a job candidates have to meet with some new requirements for their strength or endurance. So the question arises, how does this socio-economic context affect basic, biological parameters of a person and the assessment of his productivity or relevance? Is Western society moving into the phase of posthuman, in particular androgynous existence? How do educational institutions meet these challenges? [5].

Another reason for the problem to remain burning is the desire of society, in the form of the State, to determine the main vectors, principles and direction of social policy on family and demography issues. Russia was the first state in history to develop a whole range of 
measures and even the ideology of women's emancipation. Modern researchers note [6] that cultural practices (the political program started by the Bolsheviks) had the following consequences: the raise of women's authority (as a mother and a worker) in Soviet society led to the decrease of male importance. Often these practices in the USSR were formalized, for example, there was a quota for the presence of women in the Supreme Soviet of the USSR, but seats were distributed mainly between economy, agriculture and factory production sectors. Soviet women, representatives of intellectuals and managers, were not included in the Supreme Soviet. However, the development of the social sphere in the USSR, the protection of motherhood and childhood led to significant success of Russian society in solving the women's issue. Nowadays modern Russian society in the new conditions of its existence is looking back on the traditional ways of solving not only the female, but also the male issue. The male issue becomes increasingly significant for Western society in the XXI century.

The fourth reason to study gender issues in social practices in general, and in education in particular, is the unprecedented global employment situation caused by the pandemic. According to the World Happiness Report, Section 7, Work and Wellbeing During COVID-19: Impact, Inequality, Resilience, Future of work ... it is found out that, "Globally, four in ten employed women work in sectors that were hard-hit by COVID-19, including travel, retail, food, accommodation, and services".

Consequently in the nearest future education programs are expected to be streamlined in the context of surplus / lack of qualified personnel in certain sectors of the economy, which could potentially affect the ratio of representatives of both sexes in the number of students, and subsequently increase gender asymmetry in the labor market [7].

However, in the same report the following trend is described: the gender gap is getting smaller, fathers have also increased time spent on childcare and housework since the beginning of the pandemic. During the pandemic both sexes had to stay at home equally. Nevertheless it is still difficult to say whether the trend will develop in the future, but it deserves to be taken in consideration.

Studies of gender impact in education have both theoretical significance and practical relevance [8]. The development of a balanced social gender policy, as well as the assessment of its effectiveness, require scientific approach.

\section{Materials and methods}

Theoretically, this report is based on the results of empirical research carried out on the basis of Minin University. Quantitative methods (questionnaires) and qualitative methods (focus groups, in-depth interviews) are used. The first stage of studies was completed in 2017. These studies were focused on the analysis of the degree of citizens' satisfaction with the development of Russian province cultural environment (on the example of Nizhny Novgorod).

The focus on Nizhny Novgorod is determined not only by the location of the research base, but also by the status of the city in the Russian Federation. Nizhny Novgorod is a metropolis with a population of over $1,250,000$. The city itself is located in the central part of Russia, it is an industrial and cultural center. These circumstances determine high standards of cultural institutions demanded by residents and high level of critical assessments of quality of life in general.

The second stage of research is devoted to fixing and determining the functioning of gender markers in the educational environment of the university. These studies have been conducted since 2018. The object of the second stage is full-time students of universities, mainly in Nizhny Novgorod, as well as the teaching staff of higher education organizations. 
The focus on the university space was largely determined by the thesis: the university is a corporation, where an atmosphere of freedom of exchange of opinions is cultivated for the development of science and the launch of innovations, the formation of cohesion and tolerance.

Since the studentship is a group that includes representatives of various territorial communities (regional and district centers, urban-type settlements, villages), the results of the research carried out as a whole can be extrapolated to the Russian reality with some limitations. We assume that the transfer of results to the regions, primarily the North Caucasus, as well as Moscow, St. Petersburg and the Kaliningrad region, will be irrelevant.

\section{Results and discussions}

Since the focus of research interests is quite wide and changes over time, on the basis of Minin University, author's questionnaires and scenarios for qualitative research developed by a working group are used, which include both teachers and students, are used.

In the questionnaires, the questions are not grouped into blocks, but in order to achieve greater objectivity, they are arranged separately. The texts of questionnaires and interviews contain questions that are aimed at identifying the general gender identification of the respondents, establishing opinions about the initial parameters that determine the identification of a person by gender; the degree of influence of various factors and actors in the cultivation of types of masculinity and femininity in society. The central place in the questionnaires is given to questions aimed at fixing and analyzing the functioning of gender markers at the university.

The developed methods make it possible to record the opinion of the respondents about:

a) the peculiarities of male and female behavior, approved / disapproved behavior in the society;

b) the presence of gender equality / inequality in social practices in general, including in the educational environment of the university;

c) the presence of gender segregation practices at the university;

d) the sensitivity of the university environment to various manifestations of gender and gender characteristics;

e) willingness to oppose practices / maintain practices of non-equilibrium gender relations, etc.

And most importantly, the content of teaching materials allows us to explore the wellbeing of students in a gender-sensitive educational environment.

The results obtained in the course of the study, at first glance, turn out to be contradictory. The results indicate the presence of gender markers, but the reaction to their presence in the university is ambiguous.

Let us dwell in more detail on those moments that can directly testify that students feel at a gender-tinged university. Attention is drawn to the fact that the respondents almost unanimously note that the influence of gender markers in the university is less noticeable than in other spheres of life. Many respondents, noting the (relative) gender / gender neutrality of the university, compared the university with a family, school, sports organizations. Based on this comparison, individual respondents (about a tenth of the respondents) concluded that the sex and gender characteristics of a person do not matter at all at a university. Explaining their position, students and teachers most often explained this by the fact that at the university much depends on knowledge, ability to work and the desire to study / engage in scientific activity / be realized in a different way.

However, up to about $18 \%$ within the framework of quantitative methods and more than half of the interviewed students noted that they themselves faced or witnessed how gender played a special role, for example, in the formulation of an assessment: the teacher 
overestimated or underestimated the requirements for abilities (physical, mental, etc. .). Let us illustrate the thesis with an excerpt from an in-depth interview: "Sometimes the teachers ask them less [young men]. If a girl submits a written work, then they demand that everything be neat: in a file, without crumpled leaves ... and if the boys hand over, then, thank God, even though they have passed, they are already great, they don't even look at the design ... "(3rd year student Faculty of Humanities, Nizhny Novgorod). With regard to teachers, it is worth noting that, for their part, they also recognize the fact of a change in the volume of requirements based on gender, but only in specialized disciplines. For example, such a practice is recognized in the field of such training profiles as Physical Culture and Sports, Philosophy (interviewed by the Nizhny Novgorod NRD). It should be noted that the teachers, recognizing the facts of an unbalanced relationship, sought to comment on the position, for example: "How could it be otherwise? I can't ask boys and girls alike! They have different standards ... it's biology. "

It is noteworthy that the survey of students of Nizhny Novgorod universities in the 2018-2019 it is recorded the following point: $33.8 \%$ of respondents stated that they had witnessed situations where teachers discriminated against students on the basis of gender, and the most numerous cases were the facts of harassment by women teachers against girls $17.7 \% ; 8.7 \%$ of respondents pointed to harassment of girls by male teachers, the remaining percentage accounted for the facts of harassment of young men by teachers, and women teachers (4.4\%) again bypassed men. Thus, the female part of the studentship is recognized as the most discriminated group at the university, and the female part of the teaching staff is the most discriminatory. At the same time, analysis of the same questionnaire shows that $73.3 \%$ of respondents find the gender of the teacher to be unprincipled, $13.6 \%$ note that it is more comfortable for them to study with a teacher of their own gender, and $12.1 \%$ are the opposite.

It is noteworthy that any methodology aimed at identifying gender markers in the university space (the first questions of the questionnaire, interviews, throwing into the work of focus groups) initially records the answers, the content of which is the denial of gendersensitive practices. There are minimal respondents who initially state the presence of gender in the university. However, as they progress through the questionnaires, respondents designate the practices of non-equilibrium gender-based attitudes that take place within the walls of the university: jokes related to the behavior or characteristics of a particular gender, separation (independent or at the initiative of a teacher) based on gender when solving professional or educational tasks.

Analyzing the results of the study, we have to admit that even the facts of the presence of gender markers in the space of a Russian university are ascertained by the respondents with difficulty. On the one hand, a sophisticated eye could recognize in this the intent and consistent application of veiling practices. Sex / gender (here we do not distinguish between biological and social sex), indeed, refers to the fundamental codes in culture. Having undergone coding at the early stages of the formation of culture, the dichotomy of sex in Russian social practices still persists. In Russia, within the framework of the official line, 2 sexes are recognized, in this respect the concept of "gender" should rather be interpreted in the meaning of the social functions of sex, that is, as a cultural superstructure over biology. Our research shows that the perversions that exist in the "backyards" of culture while only strengthening the official line.

On the other hand, we have to admit that Russian students often simply lack so-called gender competence, which is understood as the ability to avoid being "the subject and object of situations of gender inequality". In fact, being a gender-competent person means having an understanding of discriminatory practices based on gender, having the ability not to initiate such practices, and resisting sexist influences. Our research has demonstrated the 
inability of students not only to counteract, but also to elementarily see such facts and evaluate them properly.

In this case, we could put forward the thesis that the underdevelopment of gender competence is due to the absence of relevant problems in Russian society as a whole, especially if we recall the successes of the USSR in neutralizing gender discrimination. However, when to the question: "In your opinion, are there professions / areas of activity only for men and only for women", the respondents answer in the affirmative and cite as an example pedagogy as a sphere of care that should traditionally be assigned to a woman, and what is more interesting - they recognize this as a normative practice, which they regard positively. Symptomatic in this case is not so much the fact that the respondents assigned pedagogy to women, but rather the making of a special value judgment on this matter. If the logical series is developed, a clear hierarchical ladder with rigid segregation indicators and corresponding practices is easily built.

Translating the results of our research into the category of quality of life requires us to correlate quantitative indicators with subjective assessments of the subject, and how comfortable it is for students to exist in a situation of having, for example, different levels of requirements or principles of organizing classes based on gender. Studies show that the studentship is very loyal to such things. Thus, quantitative and qualitative methods demonstrate the presence of gender markers in the university environment, while about 70 percent of research participants either determine their presence by basic, normative things about which there is no need to reflect; or they characterize it positively, explaining the facts of a non-equilibrium relationship with the concern of the state, an established tradition, a biological given. Another 20 percent "find it difficult" to assess. Thus, those who are uncomfortable under the current conditions are in a clear minority. At the same time, it should be noted that students who feel unhappy in the university space solely on the basis of gender have not been identified. However the total number of respondents is those who see discriminatory practices and feel their negative impact, the number is insignificant in comparison with other countries.

\section{Conclusion}

Thus, based on empirical data, it can be stated that the majority of Russian students feel comfortable in the gender-marked educational environment of the university. It is too early to speak about the impact of the pandemic on the gender well-being of the academic sphere - more research is needed here, and it will take time for educational institutions to react to the process. As for the socio-cultural and political contexts of the development of the topic of gender in the educational and academic environment of our country, it is absolutely necessary to take into account the influence of the traditions of each region, which ultimately determines the specifics of the Russian situation. Perhaps Western researchers would assess such a situation as a cognitive gap and an epistemological challenge, but the student community, realizing the presence of an imbalanced relationship based on gender in education, legitimizes the established practices. And since the university opens the door to an already established personality with a formed value paradigm, it would not be an exaggeration to say that not only students, but Russian reality as a whole is quite loyal to the practice of non-equilibrium gender relations.

\section{References}

1. Encyclopedia Britanica, https://www.britannica.com 
2. A. N. Zubets, Russian and international approaches to measuring the quality of life (2020)

3. R. Costanza, B. Fisher, S. Ali, C. Beer, L. Bond, R. Boumans, D. E. Gayer, Ecological Economics, 61(2-3), 267 (2007)

4. M. Castells, The Rise of the Network Society, The Information Age: Economy, Society and Culture. Oxford: Wiley-Blackwell, I (2010)

5. S. Kabisch, F. Koch; E. Urban Transformations: Sustainable Urban Development Through Resource Efficiency, Quality of Life and Resilience. Book series: Future City, 10, 1 (2018)

6. O. A. Khasbulatova, Russian Gender Policy of the 20th Century: Myths and Realities (2005)

7. M. Cotofan, J.-E. De Neve, M. Golin, M. Kaats, G. Ward, Work and Well-being during COVID-19: Impact, Inequalities, Resilience, and the Future of Work, https://worldhappiness.report

8. H. Wu; X. Ran; T. Zhang, et.al., Value in Health, 21, S227 (2018)

9. V. V. Teslinov, et.al., Substantiation of the Russian concept of lifelong education for adults (2014)

10. A. I. Subetto, Theory of quality of life (2017)

11. A. V. Babaeva, E. V. Kuznetsova, N. V. Shmeleva, M. Yu. Shlyakhov, Prospects for Science and Education, 5(41), 117 (2019) 